\title{
Vertex Turán problems for the oriented hypercube
}

\author{
Dániel Gerbner \\ Alfréd Rényi Institute of Mathematics \\ email: gerbner@renyi.hu
}

Abhishek Methuku

University of Birmingham

email: abhishekmethuku@gmail.com

Balázs Patkós

Alfréd Rényi Institute of Mathematics, Lab. of Combinatorial and Geometric Structures, Moscow Inst. of Physics and Technology

email: patkos@renyi.hu

\author{
Dániel T. Nagy \\ Alfréd Rényi Institute of Mathematics \\ email: nagydani@renyi.hu \\ Máté Vizer \\ Alfréd Rényi Institute of Mathematics \\ email: vizermate@gmail.com
}

\begin{abstract}
In this short note we consider the oriented vertex Turán problem in the hypercube: for a fixed oriented graph $\vec{F}$, determine the maximum cardinality $\operatorname{ex}_{v}\left(\overrightarrow{\mathrm{F}}, \overrightarrow{\mathrm{Q}_{n}}\right)$ of a subset $\mathrm{U}$ of the vertices of the oriented hypercube $\overrightarrow{Q_{n}}$ such that the induced subgraph $\overrightarrow{Q_{n}}[\mathrm{U}]$ does not contain any copy of $\vec{F}$. We obtain the exact value of $\operatorname{ex}_{v}\left(\overrightarrow{P_{k}}, \overrightarrow{Q_{n}}\right)$ for the directed path $\overrightarrow{P_{k}}$, the exact value of $\operatorname{ex}_{v}\left(\overrightarrow{V_{2}}, \overrightarrow{Q_{n}}\right)$ for the directed cherry $\overrightarrow{V_{2}}$ and the asymptotic value of $\operatorname{ex}_{v}\left(\vec{T}, \overrightarrow{Q_{n}}\right)$ for any directed tree $\vec{T}$.
\end{abstract}

\section{Introduction}

One of the most studied problems in extremal combinatorics is the so-called Turán problem originated in the work of Turán [15] (for a recent survey see 
[9]). A basic problem of this sort asks for the maximum possible number of edges ex $(F, G)$ in a subgraph $G^{\prime}$ of a given graph $G$ that does not contain $F$ as a subgraph.

Much less attention is paid to the vertex version of this problem. This problem can be formalized as follows: what is the the maximum cardinality $\operatorname{ex}_{v}(F, G)$, of a subset $U$ of vertices of a given graph $G$ such that $G[U]$ does not contain $F$ as a subgraph.

We will consider Turán type problems for the n-dimensional hypercube $\mathrm{Q}_{\mathrm{n}}$, the graph with vertex set $V_{n}=\{0,1\}^{n}$ corresponding to subsets of an n-element set and edges between vertices that differ in exactly one coordinate.

Edge-Turán problems in the hypercube have attracted a lot of attention. This research was initiated by Erdős [6], who conjectured that $\operatorname{ex}\left(C_{4}, Q_{n}\right)=$ $(1+\mathrm{o}(1)) n 2^{n-1}$, i.e., any subgraph of $Q_{n}$ having significantly more than half of the edges of $\mathrm{Q}_{n}$ must contain a copy of $\mathrm{C}_{4}$. This problem is still unsolved. Conlon [5] showed, extending earlier results due to Chung [3] and Füredi and Özkahya $[7,8]$, that ex $\left(C_{2 k}, Q_{n}\right)=o\left(n 2^{n}\right)$ for $k \neq 2,3,5$.

Concerning the vertex Turán problem in the hypercube $Q_{n}$, it is obvious that we can take half of the vertices of $Q_{n}$ such that they induce no edges. Kostochka [14] and later, independently, Johnson and Entringer [12] showed $\operatorname{ex}_{v}\left(C_{4}, Q_{n}\right)=\max _{j}\left\{\sum_{i \neq j} \bmod 3\left(\begin{array}{c}n \\ i\end{array}\right)\right\}$. Johnson and Talbot [11] proved a local stability version of this result. Chung, Füredi, Graham, Seymour [4] proved that if $\mathrm{U}$ contains more than $2^{\mathrm{n}-1}$ vertices, then there is a vertex of degree at least $\frac{1}{2} \log n-\frac{1}{2} \log \log n+\frac{1}{2}$ in $Q_{n}[U]$. This shows that for any star $S_{k}$ with $k$ fixed, we have $e x_{v}\left(S_{k}, Q_{n}\right)=2^{n-1}$ for large enough $n$. Alon, Krech, and Szabó [1] investigated the function $\operatorname{ex}_{v}\left(\mathrm{Q}_{\mathrm{d}}, \mathrm{Q}_{n}\right)$.

Let us note that there is a simple connection between the edge and the vertex Turán problems in the hypercube.

Proposition 1 ex $\left(F, Q_{n}\right) \leq 2^{n-1}+\frac{e x\left(F, Q_{n}\right)}{n}$.

Proof. If a subgraph $G$ of $Q_{n}$ contains more than $2^{n-1}+\frac{e x\left(F, Q_{n}\right)}{n}$ vertices, then it contains more than $\frac{\operatorname{ex}\left(F, Q_{n}\right)}{n}$ edges in every direction, thus more than $\operatorname{ex}\left(F, Q_{n}\right)$ edges altogether, hence $G$ contains a copy of $F$.

For every tree $T$, this observation implies that $\operatorname{ex}_{v}\left(T, Q_{n}\right)=\left(\frac{1}{2}+\mathcal{O}\left(\frac{1}{n}\right)\right) 2^{n}$, using the well-known result from Turán theory which states that ex $(n, T)=$ $\mathrm{O}(\mathrm{n})$ (and so $\operatorname{ex}\left(\mathrm{F}, \mathrm{Q}_{\mathrm{n}}\right)=\mathcal{O}\left(2^{\mathfrak{n}}\right)$ ). Also, together with Conlon's result on the cycles mentioned earlier, we obtain $\operatorname{ex}_{v}\left(C_{k}, Q_{n}\right)=\left(\frac{1}{2}+o(1)\right) 2^{n}$ for $k \neq 2,3,5$. 
In this paper, we consider an oriented version of this problem. There is a natural orientation of the edges of the hypercube. An edge $u v$ means that $u$ and $v$ differ in only one coordinate; if $u$ contains 1 and $v$ contains 0 in this coordinate, then we direct the edge from $v$ to $u$. We denote the hypercube $\mathrm{Q}_{\mathrm{n}}$ with this orientation by $\overrightarrow{\mathrm{Q}_{\mathrm{n}}}$. With this orientation it is natural to forbid oriented subgraphs. We will denote by $\operatorname{ex}_{v}\left(\vec{F}, \overrightarrow{Q_{n}}\right)$ the maximum number of vertices that an $\vec{F}$-free subgraph of $\overrightarrow{Q_{n}}$ can have. As vertices of the hypercube correspond to sets, instead of working with subsets of the vertices of $\vec{Q}_{n}$ we will consider families $\mathcal{G} \subseteq 2^{[n]}$ of sets. We will say that $\mathcal{G} \subseteq 2^{[n]}$ is $\overrightarrow{\mathrm{F}}$-free if for the corresponding subset $U$ of vertices of $\vec{Q}_{n}$ the induced subgraph $\vec{Q}_{n}[U]$ is $\overrightarrow{\mathrm{F}}$-free.

For example, there is only one orientation of $\mathrm{C}_{4}$ that embeds into the hypercube, we will denote it by $\overrightarrow{C_{4}}$. Hence we have $\operatorname{ex}_{v}\left(\overrightarrow{C_{4}}, \overrightarrow{Q_{n}}\right)=\operatorname{ex}_{v}\left(C_{4}, Q_{n}\right)$, which is known exactly, due to the above mentioned result of Kostochka and Johnson and Entringer. However, there are three different orientations of $\mathrm{P}_{3}$, according to how many edges go towards the middle vertex: $\overrightarrow{V_{2}}$ denotes the orientation with a source (i.e., $\overrightarrow{V_{2}}$ is the path $a b c$ such that the edge $a b$ is directed from $b$ to $a$ and the edge $b c$ is directed from $b$ to $c$ ). The directed path $\overrightarrow{P_{k}}$ is a path on $k$ vertices $v_{1}, \ldots, v_{k}$ with edges going from $v_{i}$ to $v_{i+1}$ for every $i<k$. The height of a directed graph is the length of a longest directed path in it.

If we consider the hypercube as the Boolean poset, then each edge of the hypercube goes between a set $A$ and a set $A \cup\{x\}$ for some $x \notin A$. Then in $\overrightarrow{Q_{n}}$ the corresponding directed edge goes from $A$ to $A \cup\{x\}$. A directed acyclic graph $\vec{F}$ can be considered as a poset $F$; we will say that $F$ is the poset of $\overrightarrow{\mathrm{F}}$. The poset corresponding to a directed tree is said to be a tree poset. Forbidding copies of a poset in a family of sets in this order-preserving sense has an extensive literature (see [10] for a survey on the theory of forbidden subposets). We say $\mathcal{P} \subset 2^{[n]}$ is a copy of $\mathrm{P}$ if there exists a bijection $\mathrm{f}: \mathrm{P} \rightarrow \mathcal{P}$ such that $p<p^{\prime}$ implies $f(p) \subset f\left(p^{\prime}\right)$. We say that $\mathcal{F} \subset 2^{[n]}$ is $P$-free, if there is no $\mathcal{P} \subset \mathcal{F}$ that is a copy of $P$. Observe that if $\mathrm{P}$ is the poset of the directed acyclic graph $\vec{F}$, then any P-free family is $\vec{F}$-free.

The oriented version of the vertex Turán problem in the hypercube corresponds to the following variant of the forbidden subposet problem. We say $\mathcal{P} \subset 2^{[n]}$ is a cover-preserving copy of $\mathrm{P}$ if there exists a bijection $\mathrm{f}: \mathrm{P} \rightarrow \mathcal{P}$ such that if $p$ covers $p^{\prime}$ in $P$, then $f(p)$ covers $f\left(p^{\prime}\right)$ in the Boolean poset. Thus it is not surprising that we can use techniques and results from the theory of 
forbidden subposet problems in our setting.

In this paper, we consider Vertex Turán problems for directed trees. Our main result determines the asymptotic value of the vertex Turán number $\operatorname{ex}_{v}\left(\vec{T}, \overrightarrow{Q_{n}}\right)$ for any directed tree $\vec{T}$.

Theorem 1 For any directed tree $\overrightarrow{\mathrm{T}}$ of height $\mathrm{h}$, we have

$$
\operatorname{ex}_{v}\left(\vec{T}, \overrightarrow{Q_{n}}\right)=\left(\frac{h-1}{h}+o(1)\right) 2^{n}
$$

Below we obtain the exact value of the vertex Turán number for some special directed trees (namely $\overrightarrow{\mathrm{V}_{2}}$ and $\overrightarrow{\mathrm{P}_{\mathrm{k}}}$ ).

\section{Theorem 2}

$$
\operatorname{ex}_{v}\left(\overrightarrow{V_{2}}, \overrightarrow{Q_{n}}\right)=2^{n-1}+1
$$

It would be natural to consider the following generalization of $\vec{V}_{2}$ : let $\vec{V}_{r}$ denote the star with $r$ leaves all edges oriented towards the leaves. Note that if one takes the elements of the $r$ highest levels of the Boolean poset and every other level below them, then the corresponding family in $\overrightarrow{Q_{\eta}}$ will be $\overrightarrow{V_{r}}$ free. Computing the cardinality of this family we have $\operatorname{ex}_{v}\left(\vec{V}_{r}, \overrightarrow{Q_{n}}\right)=2^{n-1}+$ $\Omega\left(n^{r-2}\right)$. We conjecture that $\operatorname{ex}_{v}\left(\vec{V}_{r}, \overrightarrow{Q_{n}}\right)=2^{n-1}+\Theta\left(n^{r-2}\right)$ holds for every $r \geq 3$.

Theorem 3 For any pair $\mathrm{k}, \mathrm{n}$ of integers with $\mathrm{k} \leq \mathrm{n}$ we have

$$
\operatorname{ex}_{v}\left(\overrightarrow{P_{k}}, \overrightarrow{Q_{n}}\right)=\max _{j \in[k]}\left\{\sum_{i \neq j \bmod k}\left(\begin{array}{l}
n \\
i
\end{array}\right)\right\} \text {. }
$$

After submitting this paper we learned that the above theorem was proved in a different context by Katona [13].

\section{Proofs}

\subsection{Proof of Theorem 1}

We follow the lines of a proof of Bukh [2] that shows that if $\mathrm{T}$ is a tree poset with $h(T)=k$ and $\mathcal{F} \subseteq 2^{[n]}$ is a T-free family of sets, then $|\mathcal{F}| \leq$ $\left(k-1+O\left(\frac{1}{n}\right)\right)\left(\begin{array}{c}n \\ \lfloor n / 2\rfloor\end{array}\right)$ holds. The proof of this theorem consists of several 
lemmas. Some of them we will state and use in their original form, some others we will state and prove in a slightly altered way so that we can apply them in our setting. First we need several definitions. For a family $\mathcal{F} \subseteq 2^{[\mathrm{n}]}$, its Lubell-function

$$
\lambda_{\mathrm{n}}(\mathcal{F})=\sum_{\mathrm{F} \in \mathcal{F}} \frac{1}{\left(\begin{array}{c}
n \\
|\mathrm{~F}|
\end{array}\right)}=\frac{1}{n !} \sum_{\mathrm{F} \in \mathcal{F}}|\mathrm{F}| !(\mathrm{n}-|\mathrm{F}|) !
$$

is the average number of sets in $\mathcal{F}$ that a maximal chain $\mathcal{C}$ in $2^{[\mathrm{n}]}$ contains. A poset $\mathrm{P}$ is called saturated if all its maximal chains have length $\mathrm{h}(\mathrm{P})$. For any poset $T$ its opposite poset $T^{\prime}$ consists of the same elements as $T$ with $t \leq T^{\prime} t^{\prime}$ if and only if $\mathrm{t}^{\prime} \leq_{\mathrm{T}} \mathrm{t}$. For a family $\mathcal{F} \subseteq 2^{[\mathrm{n}]}$ of sets, its complement family is $\overline{\mathcal{F}}=\{[\mathrm{n}] \backslash \mathrm{F}: \mathrm{F} \in \mathcal{F}\}$. Clearly, $\mathcal{F}$ contains a copy of $\mathrm{P}$ if and only if $\overline{\mathcal{F}}$ contains a copy of $\mathrm{P}^{\prime}$ and $\lambda_{\mathrm{n}}(\mathcal{F})=\lambda_{\mathrm{n}}(\overline{\mathcal{F}})$.

Lemma 1 (Bukh [2]) Every tree poset $\mathrm{T}$ is an induced subposet of a saturated tree poset $\mathrm{T}^{\prime}$ with $\mathrm{h}(\mathrm{T})=\mathrm{h}\left(\mathrm{T}^{\prime}\right)$.

An interval in a poset $\mathrm{P}$ is a set of the form $[x, y]=\{z \in P: x \leq z \leq y\}$.

Lemma 2 (Bukh [2]) If $\mathrm{T}$ is a saturated tree poset that is not a chain, then there exists $\mathrm{t} \in \mathrm{T}$ that is a leaf in $\mathrm{H}(\mathrm{T})$ and there exists an interval $\mathrm{I} \subset \mathrm{T}$ containing $\mathrm{t}$ such that $|\mathrm{I}|<\mathrm{h}(\mathrm{T})$ holds, and $\mathrm{T} \backslash \mathrm{I}$ is a saturated tree poset with $h(T)=h(T \backslash I)$.

From now on we fix a tree poset $T$ and we denote its height by $k$. We say that a chain in $2^{[n]}$ is fat if it contains $k$ members of $\mathcal{F}$.

Lemma 3 If $\mathcal{F} \subseteq \bigcup_{j=i}^{i+k-1}\left(\begin{array}{c}{[n]} \\ j\end{array}\right)$ is a family with $\lambda(\mathcal{F}) \geq(k-1+\varepsilon)$, then there are at least $(\varepsilon / \mathrm{k}) \mathrm{n}$ ! fat chains.

Proof. Let $C_{i}$ denote the number of maximal chains that contain exactly $i$ sets from $\mathcal{F}$. As $\mathcal{F} \subseteq \bigcup_{j=i}^{i+k-1}\left(\begin{array}{c}{[n]} \\ j\end{array}\right)$, we have $C_{i}=0$ for all $i>k$. Then counting the number of pairs $(F, \mathcal{C})$ with $\mathcal{C}$ being a maximal chain and $F \in \mathcal{F} \cap \mathcal{C}$, in two different ways, we obtain

$$
\sum_{i=0}^{n} i C_{i}=\lambda(\mathcal{F}) n ! \geq(k-1+\varepsilon) n ! .
$$

This, and $\sum_{i} C_{i}=n !$ imply

$$
k C_{k}=\sum_{i \geq k} i C_{i} \geq \sum_{i=0}^{n} i C_{i}-(k-1) \sum_{i<k} C_{i} \geq \varepsilon n ! .
$$


Therefore the number of fat chains in $\mathcal{F}$ is $C_{k} \geq(\varepsilon / k) n !$.

Lemma 4 Let $\mathrm{T}$ be a saturated tree poset of height $\mathrm{k}$. Suppose $\mathcal{F} \subseteq \cup_{\mathfrak{j}=i}^{i+k-1}\left(\begin{array}{c}{[n]} \\ j\end{array}\right)$ is a family with $\mathrm{n} / 4 \leq \mathrm{i} \leq 3 \mathrm{n} / 4$. Moreover, suppose $\mathcal{L}$ is a family of fat chains with $|\mathcal{L}|>\frac{4\left({ }^{(\mathrm{T} \mid+1} 2\right)}{n} \mathrm{n} !$. Then there is a copy of $\mathrm{T}$ in $\mathcal{F}$ that contains only sets that are contained in some fat chain in $\mathcal{L}$.

Proof. We proceed by induction on $|\mathrm{T}|$. If $\mathrm{T}$ is a chain, then the $\mathrm{k}$ sets in any element of $\mathcal{L}$ form a copy of $T$. In particular, it gives the base case of the induction. So suppose $T$ is not a chain. Then applying Lemma 2, there exists a leaf $t$ in $T$ and interval $I \subseteq T$ containing $t$ such that $h(T \backslash I)=k$ and $T \backslash I$ is a saturated tree poset. Our aim is to use induction to obtain a copy of $T \backslash I$ in $\mathcal{F}$ that can be extended to a copy of $T$. Finding a copy of $T \backslash I$ is immediate, but in order to be able to extend it, we need a copy satisfying some additional properties, described later.

By passing to the opposite poset $T^{\prime}$ of $T$ and considering $\overline{\mathcal{F}}$, we may assume that $t$ is a minimal element of $T$. There exists a maximal chain $C$ in $T$ that contains I, and we have $|\mathrm{C}|=k$ as $T$ is saturated. Then $s:=|C \backslash I|=k-|I| \geq 1$.

We need several definitions. Let $F_{1} \supset F_{2} \supset \cdots \supset F_{s}$ be a chain with $\left|F_{j}\right|=$ $i+k-j$ for $j=1, \ldots, s$. Then this chain is a bottleneck if there exists a family $\mathcal{S} \subset \mathcal{F}$ with $|\mathcal{S}|<|\mathrm{T}|$ such that for every fat chain $\mathrm{F}_{1} \supset \mathrm{F}_{2} \supset \cdots \supset$ $\mathrm{F}_{\mathrm{s}} \supset \mathrm{F}_{\mathrm{s}+1} \supset \cdots \supset \mathrm{F}_{\mathrm{k}}$ in $\mathcal{L}$ we have $\mathcal{S} \cap\left\{\mathrm{F}_{\mathrm{s}+1}, \ldots, \mathrm{F}_{\mathrm{k}}\right\} \neq \emptyset$. Such an $\mathcal{S}$ is a witness to the fact that $F_{1}, \ldots, F_{s}$ is a bottleneck (and we assume all sets of the witness are contained in $F_{s}$ ). We say that a fat chain is bad if its top $s$ sets form a bottleneck. A fat chain is good if it is not bad. Observe that if there is a copy $\mathcal{F}_{T \backslash I}$ of $T \backslash I$ consisting of sets of good fat chains, then we can extend $\mathcal{F}_{T \backslash I}$ to a copy of $\mathrm{T}$. Indeed, as the sets $\mathrm{F}_{1}^{\prime}, \ldots, \mathrm{F}_{\mathrm{s}}^{\prime}$ representing $\mathrm{C} \backslash \mathrm{I}$ in $\mathcal{F}_{T \backslash I}$ do not form a bottleneck and $\left|\mathcal{F}_{T \backslash I}\right|<|T|$, there must be a good fat chain $F_{1}^{\prime} \supset \cdots \supset F_{s}^{\prime} \supset F_{s+1}^{\prime} \supset \cdots \supset F_{k}^{\prime}$ such that $F_{s+1}^{\prime}, \ldots, F_{k}^{\prime} \notin \mathcal{F}_{T \backslash I}$, therefore $\mathcal{F}_{T \backslash I} \cup\left\{F_{s+1}^{\prime}, \ldots, F_{k}^{\prime}\right\}$ is a copy of $T$. Therefore all we need to prove is that there are enough good fat chains to obtain a copy of $\mathrm{T} \backslash \mathrm{I}$ by induction.

Let us bound the number of bad fat chains. If $|\mathcal{C} \cap \mathcal{F}|<s$, then $\mathcal{C}$ cannot be bad. We partition maximal chains in $2^{[n]}$ according to their sth largest set $F_{s}$ from $\mathcal{F}$. As the top $s$ sets must form a bottleneck, there is a witness $\mathcal{S}$ to this fact. This means that if $\mathcal{C}$ is bad, then $\mathcal{C}$ must meet $\mathcal{S}$ whose elements are all contained in $\mathrm{F}_{s}$. But as $|\mathcal{S}|<|\mathrm{T}|$ and all sets of $2^{\mathrm{F}_{s}} \cap \mathcal{F}$ have size between $n / 4$ and $3 n / 4$, the proportion of those chains that do meet $\mathcal{S}$ is at most $4|\mathrm{~T}| / \mathrm{n}$ (any proper non-empty subset of $F_{S}$ is contained in at most $1 /\left|F_{S}\right|$ proportion of chains going through $F_{s}$ ). This holds independently of the choice of $F_{s}$, thus 
the number of bad fat chains is at most $\frac{4|T|}{n} n$ !. So the number of good fat chains is at least

$$
|\mathcal{L}|-\frac{4|\mathrm{~T}|}{\mathrm{n}} \mathrm{n} ! \geq \frac{4\left(\left(\begin{array}{c}
|\mathrm{T}|+1 \\
2
\end{array}\right)-|\mathrm{T}|\right)}{\mathrm{n}} \mathrm{n} !=\frac{4\left(\begin{array}{c}
(\mathrm{T} \mid \\
2
\end{array}\right)}{\mathrm{n}} \mathrm{n} ! .
$$

As $|T \backslash I|<|T|$, the induction hypothesis implies the existence of a copy of $T \backslash I$ among the sets contained in good fat chains, as required.

The next lemma essentially states that if a a T-free family is contained in the union of $k$ consecutive levels, then its cardinality is asymptotically at most the cardinality of the $k-1$ largest levels. Formally, let $b(i)=b_{k, n}(i)=\max \left\{\left(\begin{array}{c}n \\ j\end{array}\right)\right.$ : $i \leq j \leq i+k-1\}$. So if $i \leq n / 2-k+1$, then $b(i)=\left(\begin{array}{c}n \\ i+k-1\end{array}\right)$, if $i \geq n / 2$, then $b(\mathfrak{i})=\left(\begin{array}{c}n \\ i\end{array}\right)$, while if $n / 2-k+1<i<n / 2$, then $b(\mathfrak{i})=\left(\begin{array}{c}n \\ \lfloor n / 2\rfloor\end{array}\right)$.

Lemma 5 If $\mathrm{T}$ is a tree poset of height $\mathrm{k}$, then there exists $\mathrm{n}_{0}$ such that for $\mathrm{n}>\mathrm{n}_{0}, \mathrm{n} / 4 \leq \mathrm{i} \leq 3 \mathrm{n} / 4-\mathrm{k}$ any $\mathcal{F} \subset \bigcup_{j=i}^{i+k-1}\left(\begin{array}{c}{[\mathrm{n}]} \\ j\end{array}\right)$ of cardinality at least $\left(k-1+\frac{k 4|T|^{2}}{n}\right) b(i)$ contains a copy of $T$.

Proof. By Lemma 1 we may suppose that $\mathrm{T}$ is a saturated tree poset. Assume $\mathcal{F} \subseteq \bigcup_{j=i}^{i+k-1}\left(\begin{array}{c}{[n]} \\ j\end{array}\right)$ is a T-free family that contains at least $\left(k-1+\frac{k 4|T|^{2}}{n}\right) b(i)$ sets. Then $\mathcal{F} \subseteq \bigcup_{j=i}^{i+k-1}\left(\begin{array}{c}{[n]} \\ j\end{array}\right)$ implies that $\lambda_{n}(\mathcal{F}) \geq k-1+\frac{k 4|T|^{2}}{n}$.

Let $\varepsilon=4 \mathrm{k}|\mathrm{T}|^{2} / \mathrm{n}$. Then we can apply Lemma 3 to find $4|\mathrm{~T}|^{2} \mathrm{n} ! / \mathrm{n}$ fat chains. Then we can apply Lemma 4 with $k=h(T)$ to obtain a copy of $T$ in $\mathcal{F}$, contradicting the $\mathrm{T}$-free property of $\mathcal{F}$.

With Lemma 5 in hand, we can now prove Theorem 1. Let us consider a $\overrightarrow{\mathrm{T}}$-free family $\mathcal{F}$. Let $\mathrm{T}$ be the poset of $\overrightarrow{\mathrm{T}}$ and let $\mathrm{T}^{*}$ be the saturated poset containing $T$ with $h(T)=h\left(T^{*}\right)=k$ - guaranteed by Lemma 1. For any integer $0 \leq i \leq n-k+1$, let $\mathcal{F}_{i}=\{F \in \mathcal{F}: i \leq|F| \leq i+k-1\}$. Observe that the $\overrightarrow{\mathrm{T}}$-free property of $\mathcal{F}$ implies that $\mathcal{F}_{i}$ is $\mathrm{T}^{*}$-free for every $i$. Note that every $\mathrm{F} \in \mathcal{F}$ belongs to exactly $\mathrm{k}$ families $\mathcal{F}_{\mathrm{i}}$ unless $|\mathrm{F}|<\mathrm{k}-1$ or $|\mathrm{F}|>\mathrm{n}-\mathrm{k}+1$. It is well-known that $\left|\left(\begin{array}{c}{[n]} \\ \leq n / 4\end{array}\right) \cup\left(\begin{array}{c}{[n]} \\ \geq 3 n / 4\end{array}\right)\right|=o\left(\frac{1}{n} 2^{n}\right)$, therefore using Lemma 5 we obtain

$$
\begin{aligned}
k|\mathcal{F}|-o\left(\frac{1}{n} 2^{n}\right) & \leq \sum_{i=n / 4}^{3 n / 4}\left|\mathcal{F}_{i}\right| \leq\left(k-1+\frac{k 4|T|^{2}}{n}\right) \sum_{i=n / 4}^{3 n / 4} b(i) \\
& \leq\left(k-1+\frac{k 4|T|^{2}}{n}\right)\left(2^{n}+k\left(\begin{array}{c}
n \\
\lfloor n / 2\rfloor
\end{array}\right)\right) .
\end{aligned}
$$


After rearranging, we get $|\mathcal{F}| \leq\left(\frac{k-1}{k}+o(1)\right) 2^{n}$.

\subsection{Proof of Theorem 2}

To prove the lower bound, we show a $\overrightarrow{V_{2}}$-free family in $\overrightarrow{Q_{n}}$ of cardinality $2^{\text {n-1 }}+1$. Simply take every second level in the hypercube starting from the $(n-1)$ st level and also take the vertex corresponding to [n].

We prove the upper bound by induction on $n$ (it is easy to see the base case $n=2)$. We will need the following simple claim.

Lemma 6 Let $\mathcal{F} \subset 2^{[n]}$ is a maximal $\overrightarrow{\mathrm{V}}_{2}$-free family, then $\mathcal{F}$ contains the set $[\mathrm{n}]$ and at least one set of size $\mathrm{n}-1$.

Proof.[Proof of lemma] First note that $[n]$ can be added to any $\vec{V}_{2}$-free family as there is only one subset of $[n]$ of size $n$. Also, if $\mathcal{F}$ does not contain any set of size $n-1$, then one such set $S$ can be added to $\mathcal{F}$. Indeed, if we add $S$, no copy of $\vec{V}_{2}$ having sets of size $n-1$ and $n$ will be created because [n] is the only set of size $n$ in $\mathcal{F} \cup\{S\}$. Furthermore, no copy of $\vec{V}_{2}$ having sets of size $n-2$ and $n-1$ will be created as $S$ is the only set of size $n-1$ in $\mathcal{F} \cup\{S\}$.

Now we are ready to prove Theorem 2 . Let $\mathcal{F} \subset 2^{[n]}$ be a $\vec{V}_{2}$-free family. For some $x \in[n]$, define

$$
\mathcal{F}_{\chi}^{-}=\{\mathrm{F} \mid \mathrm{F} \in \mathcal{F}, x \notin \mathrm{F}\} \quad \text { and } \quad \mathcal{F}_{\chi}^{+}=\{\mathrm{F} \backslash\{x\} \mid \mathrm{F} \in \mathcal{F}, x \in \mathrm{F}\} .
$$

Then $\mathcal{F}_{x}^{-}, \mathcal{F}_{x}^{+} \subset 2^{[n] \backslash\{x\}}$ and they are also $\overrightarrow{\mathrm{V}}_{2}$-free. By induction, we have

$$
|\mathcal{F}|=\left|\mathcal{F}_{x}^{-}\right|+\left|\mathcal{F}_{\chi}^{+}\right| \leq 2^{n-2}+1+2^{n-2}+1=2^{n-1}+2 .
$$

Assume that $|\mathcal{F}|=2^{n-1}+2$. Then $\left|\mathcal{F}_{x}^{-}\right|=\left|\mathcal{F}_{\chi}^{+}\right|=2^{n-2}+1$ must hold for all $x \in[n]$. By Lemma $6,\left|\mathcal{F}_{x}^{-}\right|=2^{n-2}+1$ implies that $[n] \backslash\{x\}$ and at least one set of size $n-2$ are in $\mathcal{F}$. This holds for all $x \in[n]$, so all sets of size $n-1$, and at least one set of size $n-2$ are in $\mathcal{F}$. However, these would form a forbidden $\overrightarrow{V_{2}}$ in $\mathcal{F}$, contradicting our original assumption on $\mathcal{F}$. This proves that $|\mathcal{F}| \leq 2^{\text {n-1 }}+1$.

\section{$2.3 \quad$ Proof of Theorem 3}

Let $\mathrm{U}$ be a set of vertices in $\mathrm{Q}_{\mathfrak{n}}$ such that the subgraph of $\mathrm{Q}_{\mathfrak{n}}$ induced by $\mathrm{U}$ (i.e., $\left.\mathrm{Q}_{n}[\mathrm{U}]\right)$ is $\overrightarrow{\mathrm{P}_{\mathrm{k}}}$-free. Let $\mathcal{F} \subset 2^{[\mathrm{n}]}$ be a family of subsets corresponding to $\mathrm{U}$. 
First, we will introduce a weight function. For every $\mathrm{F} \in \mathcal{F}$, let $\mathcal{w}(\mathrm{F})=\left(\begin{array}{c}\mathrm{n} \\ |\mathrm{F}|\end{array}\right)$. For a maximal chain $\mathcal{C}$, let $\mathcal{w}(\mathcal{C})=\sum_{\mathrm{F} \in \mathcal{C} \cap \mathcal{F}} \mathcal{w}(\mathrm{F})$ denote the weight of $\mathcal{C}$. Let $\mathbf{C}_{n}$ denote the set of all maximal chains in $[n]$. Then

$$
\frac{1}{n !} \sum_{\mathcal{C} \in \mathbf{C}_{n}} w(\mathcal{C})=\frac{1}{n !} \sum_{\mathcal{C} \in \mathbf{C}_{n}} \sum_{F \in \mathcal{C} \cap \mathcal{F}} w(F)=\frac{1}{n !} \sum_{F \in \mathcal{F}}|F| !(n-|F|) ! w(F)=|\mathcal{F}| .
$$

This means that the average of the weights of the full chains equals the cardinality of $\mathcal{F}$. Thus, if we can find an upper bound that is valid for the weight of any chain, then this will be an upper bound on $|\mathcal{F}|$ too.

Our assumption that there is no $\overrightarrow{P_{k}}$ means that there are no $k$ neighboring members of $\mathcal{F}$ in a chain. For a given chain $\mathcal{C}$, let $a_{1}, a_{2}, \ldots a_{t}$ denote the sizes of those elements of $\mathcal{C}$ that are not in $\mathcal{F}$. Then $0 \leq a_{1}<a_{2}<\cdots<a_{t} \leq n$, $a_{1} \leq k-1, n-k+1 \leq a_{t}$ and $a_{i+1}-a_{i} \leq k$ for all $i=1,2, \ldots t-1$. The weight of the chain $\mathcal{C}$ is

$$
w(\mathcal{C})=2^{n}-\sum_{i=1}^{t}\left(\begin{array}{l}
n \\
a_{i}
\end{array}\right) .
$$

We claim that this is maximized when the numbers $\left\{a_{1}, a_{2}, \ldots a_{t}\right\}$ are all the numbers between 0 and $n$ which give the same residue when divided by $k$.

Assume that $w(\mathcal{C})$ is maximized by a different kind of set $\left\{a_{1}, a_{2}, \ldots a_{t}\right\}$. Then there is an index $i$ such that $a_{i+1}-a_{i}<k$.

If $a_{i} \leq \frac{n}{2}$ then we can decrease the numbers $\left\{a_{1}, a_{2}, \ldots a_{i}\right\}$ by 1 . (If $a_{1}$ becomes -1 then we simply remove that number.) The resulting set of numbers will still satisfy the conditions and $w(\mathcal{C})$ increases. Otherwise, $a_{i+1}>\frac{n}{2}$ must hold. Similarly, we can increase the numbers $\left\{a_{i+1}, a_{i+2}, \ldots a_{n}\right\}$ by 1 to achieve the same result. We proved that

$$
w(\mathcal{C}) \leq 2^{n}-\min _{j \in[k]} \sum_{i \equiv j \bmod k}\left(\begin{array}{l}
n \\
i
\end{array}\right)=\max _{j \in[k]}\left\{\sum_{i \neq j \bmod k}\left(\begin{array}{l}
n \\
i
\end{array}\right)\right\}
$$

holds for any full chain $\mathcal{C}$. Therefore the same upper bound holds for $|\mathcal{F}|$ as well.

\section{Acknowledgements}

Research of D. Gerbner was supported by the János Bolyai Research Fellowship of the Hungarian Academy of Sciences and the National Research, 
Development and Innovation Office - NKFIH under the grants K 116769, FK 132060, KH 130371 and SNN 129364.

Research of A. Methuku was supported by the EPSRC, grant no. EP/ S00100X/1. The research was also partially supported by the National Research, Development and Innovation Office NKFIH, grants K116769, K132696, and by the Institute for Basic Science (IBS-R029-C1).

Research of D.T. Nagy was supported by the National Research, Development and Innovation Office - NKFIH under the grants K 116769, K 132696 and FK 132060.

Research of B. Patkós was supported by the grant of Russian Government N 075-15-2019-1926 and by the National Research, Development and Innovation Office - NKFIH under the grants SNN 129364 and FK 132060.

Research of M. Vizer was supported by the Hungarian National Research, Development and Innovation Office - NKFIH under the grant SNN 129364, KH 130371 and KF 132060, by the János Bolyai Research Fellowship of the Hungarian Academy of Sciences and by the New National Excellence Program under the grant number UNKP-19-4-BME-287.

All of the authors were funded by the Taiwanese-Hungarian Mobility Program of the Hungarian Academy of Sciences.

\section{References}

[1] N. Alon, A. Krech, and T. Szabó, Turán's theorem in the hypercube, SIAM Journal on Discrete Mathematics, 21(1):66-72, 2007.

[2] B. Bukh, Set families with a forbidden subposet, The Electronic Journal of Combinatorics, 16(1):142, 2009.

[3] F. R. Chung, Subgraphs of a hypercube containing no small even cycles, Journal of Graph Theory, 16(3):273-286, 1992.

[4] F. R. Chung, Z. Füredi, R. L. Graham, and P. Seymour, On induced subgraphs of the cube, Journal of Combinatorial Theory, Series A, 49(1):180-187, 1988.

[5] D. Conlon, An extremal theorem in the hypercube, The Electronic Journal of Combinatorics, 17(1):R111, 2010.

[6] P. Erdős, On some problems in graph theory, combinatorial analysis and combinatorial number theory, Graph Theory and Combinatorics (Cambridge, 1983), Academic Press, London, pages 1-17, 1984. 
[7] Z. Füredi and L. Özkahya, On 14-cycle-free subgraphs of the hypercube, Combinatorics, Probability \& Computing, 18(5):725, 2009.

[8] Z. Füredi and L. Özkahya, On even-cycle-free subgraphs of the hypercube, Journal of Combinatorial Theory Series A, 118(6):1816-1819, 2011.

[9] Z. Füredi and M. Simonovits, The history of degenerate (bipartite) extremal graph problems. In Erdös Centennial, pages 169-264. Springer, 2013 .

[10] J. R. Griggs and W.-T. Li. Progress on poset-free families of subsets. in: Recent Trends in Combinatorics, pages 317-338, 2016.

[11] J. R. Johnson and J. Talbot. Vertex Turán problems in the hypercube. Journal of Combinatorial Theory, Series A, 117(4):454-465, 2010.

[12] K. A. Johnson and R. Entringer, Largest induced subgraphs of the ncube that contain no 4-cycles, Journal of Combinatorial Theory, Series $B, 46(3): 346-355,1989$.

[13] G. O. H. Katona, Families of subsets having no subset containing another one with small difference, Nieuw Arch. Wiskunde, 20(3):54-67, 1972.

[14] E. Kostochka, Piercing the edges of the n-dimensional unit cube, Diskret. Analiz Vyp, 28(223):55-64, 1976.

[15] P. Turán, On an external problem in graph theory, Mat. Fiz. Lapok, 48:436-452, 1941. 